\title{
LEAFY HEAD2, which encodes a putative RNA-binding protein, regulates shoot development of rice
}

\author{
Guo Sheng Xiong ${ }^{1,3, *}$, Xing Ming $\mathrm{Hu}^{2, *}$, Yong Qing Jiao ${ }^{1}$, Yan Chun $\mathrm{Yu}^{1}$, Cheng Cai Chu ${ }^{1}$, Jia Yang $\mathrm{Li}^{1}$, \\ Qian Qian', Yong Hong Wang ${ }^{1}$
}

${ }^{I}$ State Key Laboratory of Plant Genomics and National Center for Plant Gene Research, Institute of Genetics and Developmental Biology, Chinese Academy of Sciences, Beijing 100101, China; ${ }^{2}$ State Key Laboratory of Rice Biology, China National Rice Research Institute, Chinese Academy of Agricultural Sciences, Hangzhou 310006, China; ${ }^{3}$ Graduate University of Chinese Academy of Sciences, Beijing 100049, China

During vegetative development, higher plants continuously form new leaves in regular spatial and temporal patterns. Mutants with abnormal leaf developmental patterns not only provide a great insight into understanding the regulatory mechanism of plant architecture, but also enrich the ways to its modification by which crop yield could be improved. Here, we reported the characterization of the rice leafy-head2 (lhd2) mutant that exhibits shortened plastochron, dwarfism, reduced tiller number, and failure of phase transition from vegetative to reproductive growth. Anatomical and histological study revealed that the rapid emergence of leaves in $l h d 2$ was resulted from the rapid initiation of leaf primordia whereas the reduced tiller number was a consequence of the suppression of the tiller bud outgrowth. The molecular and genetic analysis showed that $L H D 2$ encodes a putative RNA binding protein with $67 \%$ similarity to maize TE1. Comparison of genome-scale expression profiles between wild-type and $l h d 2$ plants suggested that $L H D 2$ may regulate rice shoot development through $K N O X$ and hormone-related genes. The similar phenotypes caused by LHD2 mutation and the conserved expression pattern of $L H D 2$ indicated a conserved mechanism in controlling the temporal leaf initiation in grass.

Cell Research (2006) 16:267-276. doi:10.1038/sj.cr.7310034; published online 16 March 2006

Keywords: phyllotaxy, plastochron, LHD2, RNA-binding protein, stem elongation, plant architecture, Oryza sativa L

\section{Introduction}

The diversity of plant body elaborates mainly through the post-embryonic development of aerial lateral organs, which are ultimately derived from franks of a group of selfrenewal stem cells within shoot apical meristem (SAM). An indeterminate ground cellular state in meristem that allows cells in SAM to either acquire stem cell identity or be recruited into organ primordia was specified by

\footnotetext{
"These two authors contributed equally to this work Correspondence: Yong Hong Wang ${ }^{1}$, Qian Qian ${ }^{2}$

${ }^{1}$ Tel: 86-10-64889377; Fax: 86-10-64873428;

Email: yhwang@genetics.ac.cn

${ }^{2}$ Email: qianqian188@hotmail.com

Received 26 Jan 2006; revised 10 Feb 2006; accepted 14 Feb 2006; published online 16 March 2006
}

maintaining a high cytokinin and low GA ratio that directly regulated by the KNOTTED-like Homeobox $(K N O X)$ genes [1-4]. Lateral organogenesis involves the recruitment of founder cells and their transition from an indeterminate cellular state to a determinate fate [2]. Initiation of the leaf organogenesis is marked by the down-regulation of $K N O X$ expression in a subset of cells in the periphery of the SAM and activation of genes including $P H A N, B O P$ and $Y A B B Y$ families that maintain the repression of $K N O X$ expression and the regulation of leaf polarity during leaf development $[2,3,5]$. During vegetative developmental stage, the SAM continues to produce leaf primordia in a regular pattern over space (phyllotaxy) and time (plastochron) [6]. The field inhibitory hypothesis is applauded to explain plant phyllotaxy, proposing that the new primordia will form only after escaping the biochemical constraint made by the existing 
primordia [7]. Recent works revealed that the biochemical constraints could be established by polar auxin transport [8-10]. The initiation of lateral organ primordia is induced by a local auxin maximum accumulated in the peripheral zone of SAM, then next primodium formation may initiate at the site most distant to the preexisting primordium because the established primordia act as a sink to deplete auxin accumulation within surrounding cells [7-10]. Since changes in size and/or organization of SAM can alter the field in which auxin acts, a number of mutants exhibit close association of abnormal phyllotaxy with modified meristems [11-14]. The maize abphyll mutant has an enlarged SAM with abnormal phyllotaxy [13]. Molecular analysis of abphyll has revealed that ABPHYL1 encodes a cytokinin inducible response regulator, which controls the phyllotactic pattern through negative regulation of the expanding shoot meristems $[13,15]$. Rice shoot organi- zation (sho) and Arabidopsis altered meristem program 1 (ampl) have malformed SAMs and show irregular patterns of phyllotaxy and plastochron $[11,14]$. It may have coordination between temporal and spatial regulation of leaf development, because the disruption in phyllotaxy simultaneously affects plastochron in these mutants. It is possible that the inhibition effect of preexisting primodia may not only determine the site of leaf initiation, but also prevent the precocious development of new leaves [7, 16]. However, the analysis of the rice plastochron 1 mutant that shows decreased plastochron with normal phyllotaxy provided the insight of the disassociation between regulatory pathway governing spatial and temporal patterns of the leaf initiation $[12,17]$. Molecular analysis revealed that PLA1 encodes CYP78A11, a member of cytochrome P450 family involved in phytohormone biosynthetic pathways [17]. Either exogenous application of gibberellic acid (GA) or

Table 1 List of the Primers used and molecular markers developed in this study

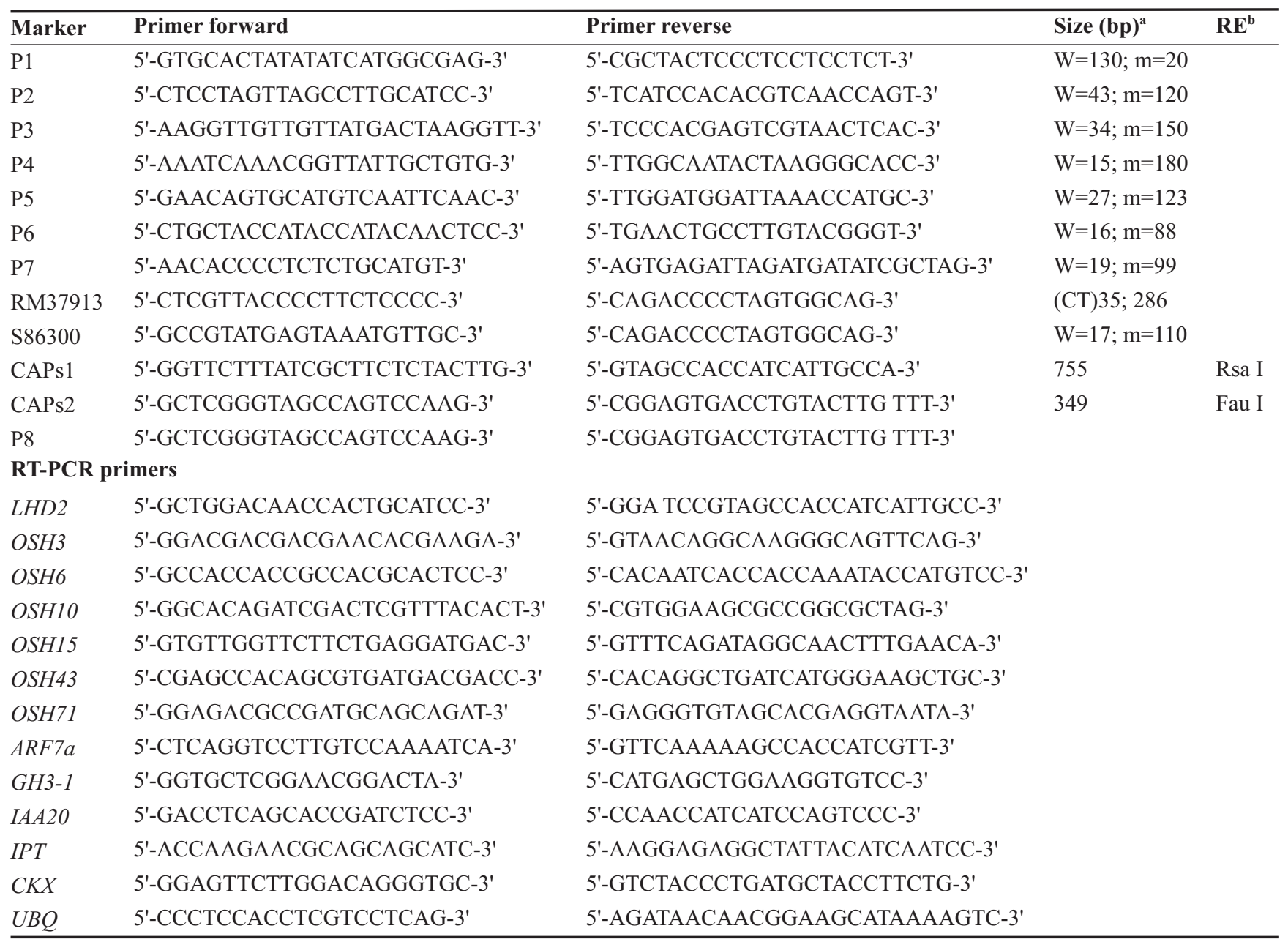

${ }^{\mathrm{a}} \mathrm{W}$ refers to the fragment size for the wild type and $\mathrm{m}$ for $l \mathrm{hd} 2-1$.

${ }^{b} \mathrm{RE}$ refers to the restriction enzymes used in this study. 
inhibition of auxin transport results in changes in phyllotaxy and plastochron, which suggests that phytohormones may regulate both plastochron and phyllotaxy [17-19].

An alternative mechanism that may regulate the timing and spacing of the leaf initiation comes from the identification of maize TERMINAL EAR1 (TE1), which encodes a putative RNA-binding protein [20]. The te1 mutant plant initiates leaves more frequently and exhibits an irregular phyllotaxy in addition to abnormal internode length. Transcripts of TE 1 accumulate in a semicircular ring embracing sites of leaf initiation that aligned with leaf midrib [20,21]. The close correlation between the TE1 expression pattern and defects in leaf initiation suggests that the RNA binding protein functions in regulating leaf initiation $[16,20]$.

Manipulation of plant architecture is thought to be an important way to increase crop yield [22, 23]. Isolation of genes regulating plastochron is of agronomical importance, because the number of leaves affects the number of tillers thus determines the number of panicles [17, 24]. We here report the characterization of the rice mutant $l h d 2$ and the molecular cloning of the LHD2 gene. The $l h d 2$ mutant exhibits multiple phenotypes including dwarfism, abnormal plastochron, reduced tiller number, and failure of panicle generation, which is similar to the previously identified mutant $l h d[25,26]$. Molecular analysis showed that LHD2 is a functional homologue of maize TE1. Genome-scale expression profile analysis suggested that $L H D 2$ may play essential roles in regulating plant architecture by interacting with plant hormones and homeobox genes.

\section{Materials and Methods}

\section{Plant materials and growth condition}

The rice (Oryza sativa L.) lhd2-1 and lhd2-3 were spontaneous mutants of YunDao32 (japonica) and TN1 (indica), and lhd2-2 was isolated from Nipponbare (japonica) mutagenized with ethyl methanesulfonate (EMS). Rice plants were cultivated in the experimental field at the China National Rice Research Institute in Hangzhou in the natural growing seasons.

$R N A$ extraction and reverse transcription-polymerase chain reaction ( $R T-P C R)$

Total RNA was isolated from $14 \mathrm{~d}$ wild-type and $l h d 2$ plants by a guanidine thiocyanate extraction method as previously described [27]. To conduct RT-PCR analysis, cDNA strands are synthesized as previously described [22]. $1.0 \mu \mathrm{g}$ product was subsequently used to amplify the target genes. Primers used in expression pattern analysis were listed in Table 1.

\section{Scanning electron microscopy}

Samples were prepared as described previously [28] with some modifications. Briefly, samples were fixed with $2.5 \%(\mathrm{v} / \mathrm{v})$ glutaraldehyde in $0.1 \mathrm{M}$ sodium phosphate buffer $(\mathrm{pH} 7.2)$ and incubated at $4{ }^{\circ} \mathrm{C}$ overnight. After being rinsed with $0.1 \mathrm{M}$ sodium phosphate buffer, they were post-fixed in $1 \%(\mathrm{w} / \mathrm{v})$ osmium tetroxide for $2 \mathrm{~h}$ at
$4{ }^{\circ} \mathrm{C}$ and rinsed with the same buffer. Samples were then dehydrated in a graded series of ethanol. For scanning electron microscopy, $100 \%$ ethanol was replaced with 3-methylbutyl acetate. Samples were critical-point dried, sputter-coated with platinum, and observed under a scanning electron microscope (model S-570; Hitachi, Tokyo, Japan).

\section{Histological analysis}

Samples were fixed with the formalin-acetic acid-alcohol (FAA) fixative solution at $4{ }^{\circ} \mathrm{C}$ overnight followed by dehydration steps and then embedded in paraffin (Paraplast Plus, Sigma). The tissues were sliced into $8 \mu \mathrm{m}$ and dried overnight onto 3-amino-propyltriethoxy silanecoated slides (ProbeOn Plus, Fisher Biotech Co.). Sections were stained with Safranin O and Fast Green (Fisher Biotech Co.) and observed under bright-field through a microscope (Leica DMR) and photographed using a Micro Color Charge-coupled Device (CCD) camera (Apogee Instruments).

\section{Map-based cloning of LHD2}

An $\mathrm{F}_{2}$ mapping population was generated by a cross between the
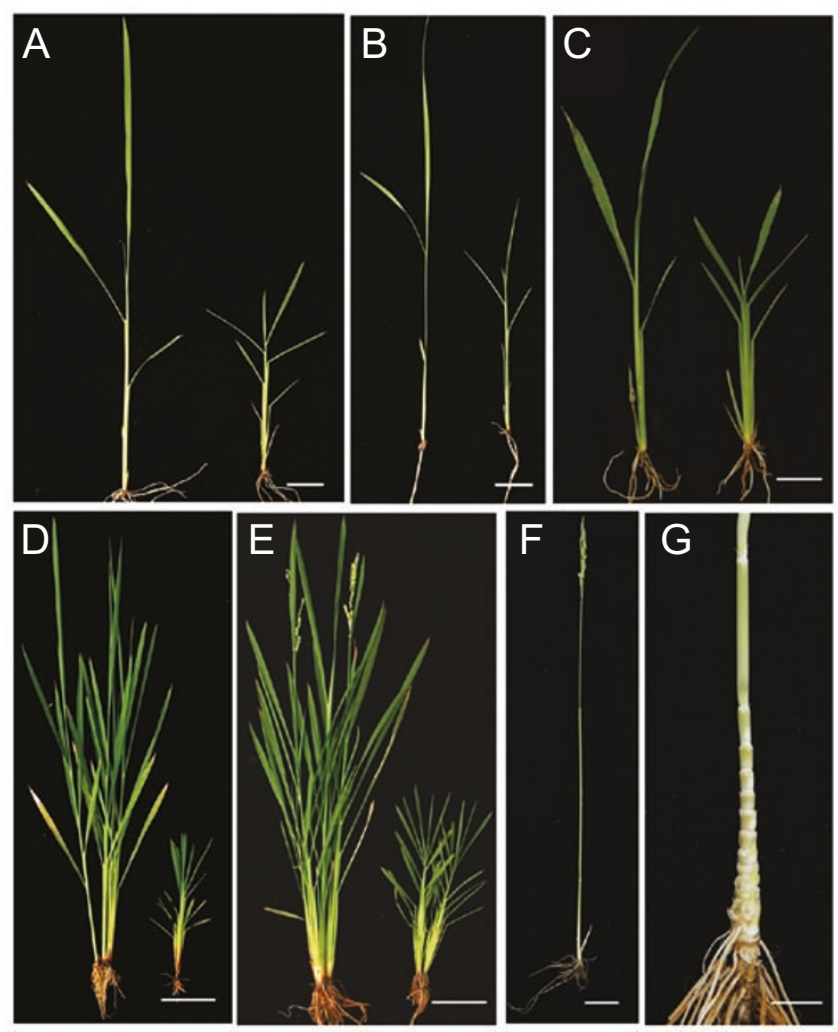

Figure 1 Phenotype of the $l h d 2$ mutant plant. (A) $14 \mathrm{~d}$ seedling of wild type (left) and lhd2-1 (right). (B) $14 \mathrm{~d}$ seedling of wild type (left) and $l h d 2-2$ (right). (C) $14 \mathrm{~d}$ seedling of wild type (left) and lhd2-3 (right). (D) Wild-type (left) and lhd2-1 (right) plants at the tillering stage. (E) Wild-type (left) and $l h d 2-1$ (right) plants at the heading stage. (F) and (G) The stems of wild-type and $l h d 2-1$ plants at the heading stage. Bars in $\mathbf{A}-\mathbf{B}=1 \mathrm{~cm}$, in $\mathbf{C}=2 \mathrm{~cm}$, in $\mathbf{D}-\mathbf{F}=5$ $\mathrm{cm}$, and in $\mathbf{G}=1 \mathrm{~cm}$. 
LHD2-1/lhd2-1 heterozygote and MingHui63, a polymorphic indica variety, and the rice genomic DNA was prepared as described [28]. The LHD2 locus was diagnostically mapped between SSRs markers RM1361 and RM104 on Chromosome 1 using $36 \mathrm{~F}_{2}$ plants of lhd 2-1 homozygotes, further placed into the DNA fragment between the markers RM37913 and S86300 using $1080 \mathrm{~F}_{2}$ lhd2-1 mutant plants, and finally placed in an interval of a $\sim 40 \mathrm{~kb}$ DNA fragment between the P3 and P5 markers and co-segregated with the P4 marker, which were developed in this work (Table 1). To sequence the $l h d 2-1, l h d 2-2$ and $l h d 2-3$ alleles, the entire genomic regions were amplified from each allele and its corresponding wild-type plant by PCR with LA-Taq (TaKaRa, Dalian, China). The mutations in $l h d 2-1$, lhd 2-2 and $l h d 2-3$ were identified by directly sequencing the PCR products. To verify the mutation in $l h d 2$ mutant alleles, CAPs 1, CAPs2 and P8 (Table 1) were developed for $l h d 2-1$, $l$ hd $2-2$ and $l h d 2-3$, respectively.

\section{Results}

\section{Morphological characterization of the lhd 2 mutant}

To understand the mechanism underlying the genetic control of plant architecture, we have collected several types of mutants altered in the overall plant body plan. Among them, three mutants showing the similar phenotypes to the leafy-head $(l h d)$ mutants $[25,26]$ were isolated from japonica varieties of Yundao32 and Nipponbare and an indica variety of $T N 1$, respectively. These mutants exhibited dwarfism, shortened plastochron, and a prolonged vegetative developmental stage (Figure 1). Genetic complementation test revealed that the three mutants are allelic (data not shown) and therefore were designated as $l h d 2-1$, lhd 2-2 and $l h d 2-3$, respectively. The $l h d 2-1$ mutant was representatively used for the further study in this work.

At the seedling stage, $l h d 2$ plants could be distinguished from the wild type by the rapid emergence of leaves with reduced leaf size and plant height (Figure 1A to 1C). Detailed phenotypic observation revealed that the mutant plants produced less tillers than the wild type at the tillering stage (Figure 1D) and could not form any panicle at the heading stage (Figure 1E).

To find out the role of $L H D 2$, we characterized the $l h d 2-1$ mutant at both anatomical and histological levels. In the longitudinal sections through apices of $14 \mathrm{~d}$ seedlings, more leaf primordia and tiller buds could be observed in $l h d 2-1$ than that in wild-type plants (Figure 2A, 2D), indicating that the rapid emergence of leaves resulted from the rapid initiation of leaf primordia and the reduced tiller number in the $l h d 2-1$ seedling was a consequence of the suppression of the tiller bud outgrowth other than the defects in tiller bud initiation. Furthermore, the $l h d 2-1$ intercalary meristem was apparently enlarged (Figure 2F) compared with that of the wild type (Figure $2 \mathrm{E}$ ), producing many more nodes in the lhd 2-1 mature plant (Figure 1F and 1G). Nevertheless, the elongation of the internodes was inhibited in $l h d 2-1$ (Figure
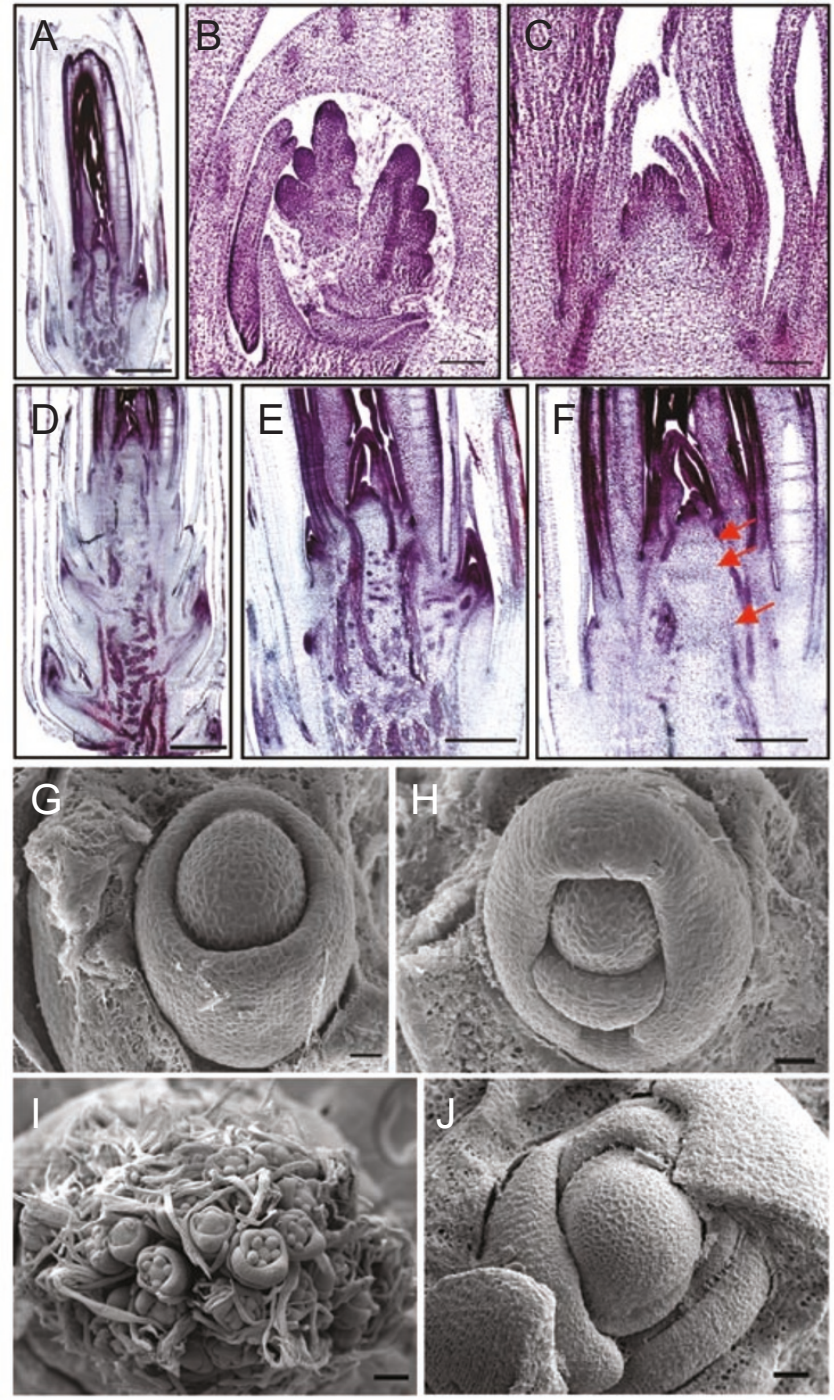

Figure 2 Structure of the lhd 2 SAM and stem. (A) Longitudinal section of the14d wild-type shoot apex. (B) Longitudinal section of the wild-type reproductive SAM. (C) Longitudinal section of the lhd2-1 SAM, indicating the block of phase transition from vegetative to reproductive growth. (D) Longitudinal section of the14d lhd2-l shoot apex. (E) Structure of the 14d wild-type stem in which cells and vascular bundles were randomly arranged, but the node and internode were not differentiated. (F) Structure of the $14 \mathrm{~d}$ lhd2-1 stem in which the node and internode were clearly differentiated. Arrows in (F) indicate the position of the nodal region. (G) SEM view of the wild-type vegetative SAM. (H) SEM view of the $l$ hd $2-1$ vegetative SAM. (I) SEM view of the wild-type reproductive SAM. (J) SEM view of the $l h d 2-1$ SAM, indicating the block of the phase transition from vegetative to reproductive growth. Scale bars in (A)-(I) $=100$ $\mu \mathrm{m}$, and in $(\mathbf{J})=20 \mu \mathrm{m}$.

$1 \mathrm{G})$, which resulted in a dwarf phenotype. Additionally, the $l h d 2$ mutation also affected the phase transition from 
the vegetative to reproductive growth. Scanning electron microscope (SEM) observation revealed that no significant morphological difference could be found between $l h d 2-1$ and the wild-type SAMs in the seedling apices (Figure $2 \mathrm{G}$ and $2 \mathrm{H}$ ). However, while the wild-type SAM underwent the transition from the vegetative to reproductive phase to generate rachis branches (Figure 2B and 2I), the $l h d 2$ SAM failed in phase transition and maintained the vegetative identity to produce many more leaves than the wild type (Figure 2C and 2J). These results suggest that LHD2 may play multiple roles in the initiation of leaf primordia, elongation of internodes, outgrowth of tiller buds and the phase transition.

\section{Molecular cloning of LHD2}

To map the LHD2 locus, we generated a $\mathrm{F}_{2}$ mapping population derived from a cross between the LHD2-1/ lhd2-1 heterozygote and Minghui63, a polymorphic indica variety. Linkage analysis of $36 \mathrm{~F}_{2}$ plants that showed the lhd2-1 mutant phenotype primarily placed the LHD2 locus

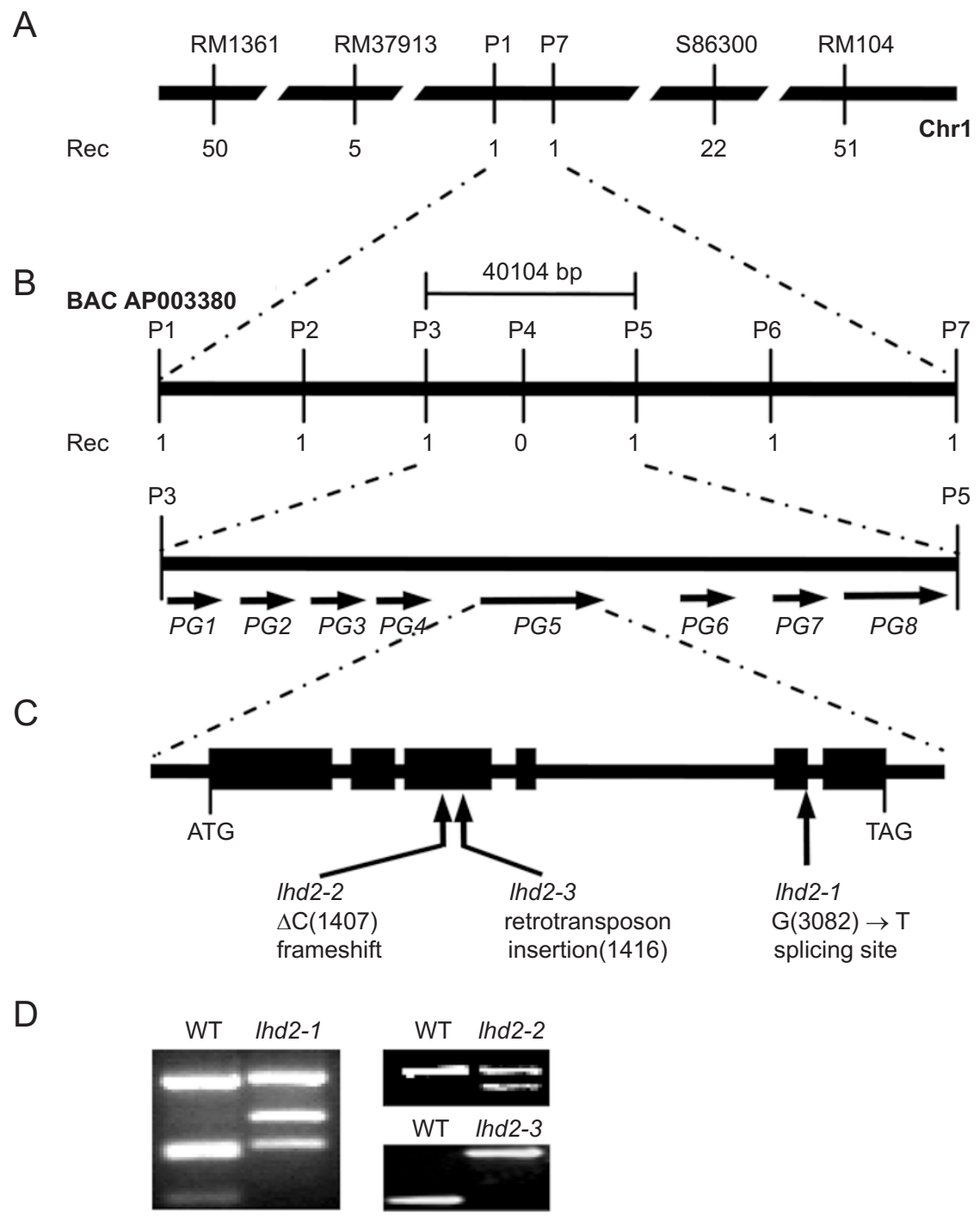

Figure 3 Positional cloning of LHD2. (A) The LHD2 locus was mapped in the Chromosome 1 (Chr 1) between SSRs markers RM1361 and RM104. The numerals indicate the number of recombinants (Rec) identified from 1080 F2 mutant plants. (B) Fine mapping of the LHD2 locus with the markers (P1 to P7) developed based on the sequence of BAC clone AP003380. The LHD2 locus was narrowed to a $\sim 40 \mathrm{~kb}$ genomic DNA region between markers P3 and P5 and cosegregated with marker P4. PG1, B1417F08.22; PG2, B1417F08.23; PG3, B1417F08.25; PG4, B1417F08.26; PG5, B1417F08.27; PG6, B1417F08.29; PG7, B1417F08.30; PG8, B1417F08.31. (C) The LHD2 structure, showing the mutated sites of the three $l$ hd 2 alleles. The start codon (ATG) and the stop codon (TGA) are indicated. Closed boxes indicate the coding sequence and lines between boxes indicate introns. (D) Molecular identification of the mutations of $l h d 2-1, l h d 2-2$ and $l h d 2-3$ by markers CAPs1, CAPs 2 and P8, as indicated in Table 1. 


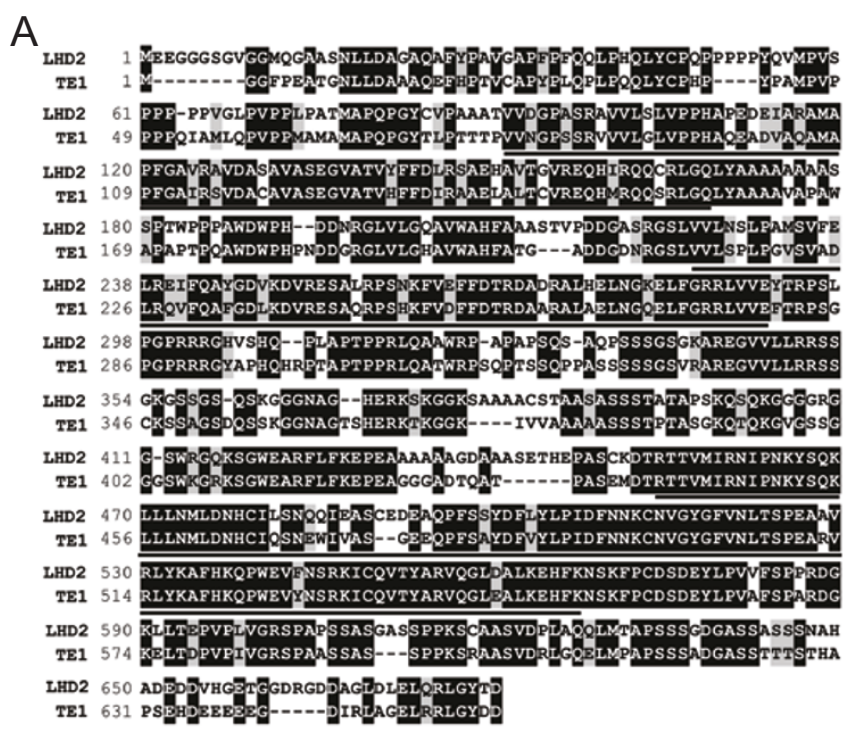

B

1 ATGGAGGAAGGAGGTGGGAGTGGGTGGGTGGGATGCAGGGAGCGGCGTCGAATCTTCTG

121 CCGCACCAGCTGTACTGCCCGCAGCCGCCGCCGCCGCCGTACCAGGTCATGCCGGTGTCG

181 CCGCCGCCGCCGCCGGTGGGTTTGCCTGTGCCGCCGCTGCCGGCGACGATGGCGCCGCAG

CCGCCGCCGCCGCCGGTGGTTTGCTTTGCCGCCGCTGCCGGCGACGATGGCGCCGCAG

CCGGGCTACTGCGTGCCGGCGGCCGCGACGGTGGTGGACGGTCCGGCCAGCCGCGCCGTC TTCGGTGCGGTGCGCCCGTGGACGCGTCGGCGGTGGCGTCCGAGGGCGTCGCGACCGTC TACTTCTTCGATCTCCGCTCCGCCGAGCACGCCGTCACGGGGGTCCGCGAGCAGCACATC TACTTCTTCGATCTCCGCTCCGCCGAGCACGCCGTCACGGGGGTCCGCGAGCAGCACATC CCGACCTGGCCCCCGCCTGCGTGGGACTGGCCCCACGACGACAACCGCGGGCTCGTCCTC GGCCAGGCCGTCTGGGCCCACTTCGCCGCCGCCTCCACCGTCCCCGACGACGGCGCCAGC CGCGGCTCCCTCGTCGTGCTCAATTCCCTCCCCGCCATGTCCGTGTTCGAACTCCGCGAA ATCTTCCAAGCATACGGTGACGTGAAGGACGTGAGGGAGTCGGCGCTGCGGCGAGCAAC AAGTTCGTCGAGTTCTTCGACACGCGCGACGCCGACCGCGCGCTCCACGAGCTCAACGGC AAGGAGCTCTTCGGCCGCCGCCTCGTCGTCGAGTACACGCGCCCTTCCCTCCCCGGCCCA CGCAGGCGCGGGCACGTGTCGCACCAGCCCTTGGCCCCGACGCCGCCGAGGCTGCAGGCG GCTTGGCGGCCGGCGCCGGCGCCGTCGCAGTCTGCGCAGCCGTCGTCGTCTGGCTCCGGC AAGGCGAGGGAAGGCGTGGTGCTTCTGCGCAGGAGCTCCGGGAAAGGTAGCTCGGGTAGC CAGTCCAAGGGCGTGGCAATGCTGGCCACGAACGGAAGAGCAAGGGCGGCAAGAGCCC GCGGCGGCGTGTTCGACGGCGGCTTCAGCATCGTCGTCTACCGCAACGGCGCCCAGCAAG CAAAGCCAGAAAGGCGGCGGCGGCGTGGCGGGAGCTGGAGAGGCCAGAAGAGCGGGTGG $\triangle$ GGCGGCGGC

GAGGCTCGCTTCCTGTTCAAAGAACCCGAGGCCGCGGCCGCCCCGCCGGCGACGCTGCC

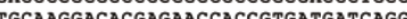
AACATCCCAAACAAGTACAGCCAGAAGCTACTGCTCAACATGCTGGACAACCACTGCATC

CTCTCCAACCAGCAGATCGAGGCGAGCTGCGAAGACGAAGCCCAGCCATTCTCCTCCTAC GATTCCTCTACCTCCCCATAGATTTCAacaAcaAgtgCaACGTGGGCTATGGCTTCGTC

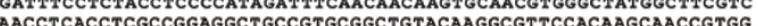
GAGGACHCGCGGGCTGCGGG

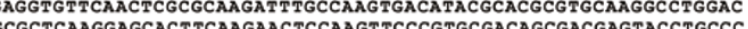
GCGCTCAAGGAGCACT TCAAGAACTCCAAGTTCCCGTGCGACAGCGACGAGTACCTGCCC CGCTCGCCGGCACCGTCGTCGGCGTCCGGGGCGTCGTCGCCGCCCAAGAGCTGCGCCGCG AGCGTCGACCCACTCGCGCAGCAGCTCATGACAGCGCCGTCTTCCTCCGGCGACGGCGC

TCCTCCGCCTCCTCGTCCAATGCCCACGCCGACGAGGATGACGTCCATGGCGAAACCGGT TAGGACCGTGGCGACGACGCGGGGCTCGATCTGGAGCTACAGCGCCTAGGCTACACTGAC TAG

Figure 4 Sequence alignment. (A) Alignment of rice LHD2 and maize TE1. Numbers at left refer to the positions of amino acid and the conserved RRM motifs were underlined. (B) Comparison of the cDNA sequences between subspecies of indica and japonica. Numbers at left refer to the positions of nucleotide. Red letters stand for the polymorphic nucleotides in japonica, green for the polymorphic nucleotides that cause amino acid changes in japonica, and green triangle for the inserted nucleotides in japonica.

in an interval between SSRs markers RM1361 and RM104 on Chromosome 1 (Figure 3A). To fine-map LHD2, 1080 $\mathrm{F}_{2}$ mutant plants were analyzed using 7 newly developed PCR-based markers, P1 to P7 (Table 1 and Figure 3B). LHD2 was pin-pointed within an interval of $\sim 40 \mathrm{~kb}$ DNA fragment between the $\mathrm{P} 3$ and $\mathrm{P} 5$ markers and co-segregated with the P4 marker in the BAC clone AP003380. Within this region, there are eight predicted genes, $P G 1$ to $P G 8$. Sequencing these genes in the $l h d 2-1$ allele revealed a Gto-T point mutation at the nucleotide 3082 in $P G 5$, which leads to a splicing error (Figure 3C). No mutation could be found in the other predicted genes. Mutations were also identified in PG5 in the other $l h d 2$ alleles. In $l h d 2-2$, a single nucleotide $\mathrm{C}$ was deleted in the third exon at the nucleotide 1407 , which causes a frameshift and produces a premature translational product (Figure $3 \mathrm{C}$ ). In $l h d 2-3$, the $1972 \mathrm{bp}$ retrotransposon was inserted in the third exon at the nucleotide 1416 , which results in a premature translational product. These three mutations were also confirmed by molecular markers developed from the sequences of mutated LHD2 in $l h d 2-1$, lhd $2-2$ and $l h d 2-3$, respectively (Table 1 and Figure 3D). Moreover, a database search with the LHD2 sequence demonstrated that LHD2 shared the highest sequence similarity to maize $T E 1$ [20] that en- codes a protein containing putative RNA recognition motif (RRM) (Figure 4A). Sequence comparison between indica and japonica subspecies revealed only $18 \mathrm{bp}$ differences at the genomic DNA level that caused only 5 changes at the protein level (Figure 4B). Loss-of-function mutation in maize $T E 1$ causes an increase in leaf initiation, an irregular phyllotaxy and an altered internode length [20]. Taken all this together, we concluded that LHD2 (accession number: DQ393277), the rice orthorlog of maize TE1, is the gene responsible for the phenotype of $l h d 2$.

\section{The expression pattern of LHD2}

To examine the expression pattern of LHD2, we conducted a semi-quantitative RT-PCR analysis using total RNA isolated from different organs. As shown in Figure 5A, LHD2 was expressed mainly in the shoot apex region. No expression could be detected in roots, nodes, internodes, leaves, and leaf sheaths. This organ-specific expression pattern is consistent with the action of LHD2 that is required for the shoot development of rice plant.

The altered expression patterns of KNOX and phytohormone-related genes in 1 hd 2

To investigate the molecular mechanism of LHD2 in 
Table 2 Differentially expressed genes identified by microarray analysis

\begin{tabular}{|c|c|c|}
\hline Coded protein/putative function & OGI $^{\mathrm{a}}$ & $\mathbf{E R}^{\mathbf{b}}$ \\
\hline \multicolumn{3}{|l|}{ Transcription factors } \\
\hline AUX/IAA family & Os02g56120 & -2.4 \\
\hline Auxin response factor 16 & Os01g13520 & -1.5 \\
\hline Auxin response factor $7 \mathrm{a}$ & Os06g48950 & -1.2 \\
\hline AP2 domain transcription factor, putative & Os01g73770 & 3 \\
\hline AP2 domain transcription factor, putative & Os08g36920 & 3 \\
\hline AP2 domain transcription factor, putative & Os03g08460 & 1.3 \\
\hline AP2 domain transcription factor, putative & Os01g46870 & 1.2 \\
\hline AP2 domain transcription factor, putative & Os03g12950 & 1.1 \\
\hline AP2 domain transcription factor, putative & Os03g09170 & -2 \\
\hline Dof domain transcription factor & Os07g48570 & -1.7 \\
\hline Helix-loop-helix DNA-binding domain & Os01g39330 & 1.5 \\
\hline Helix-loop-helix DNA-binding domain & Os08g39630 & -1.2 \\
\hline Helix-loop-helix DNA-binding domain & Os09g32510 & -1.3 \\
\hline Helix-loop-helix DNA-binding domain & Os $10 \mathrm{~g} 23050$ & -1.6 \\
\hline KNOTTED-1-like homeobox protein & Os03g47020 & 1.1 \\
\hline KNOX2 domain, putative & Os07g03770 & -1.3 \\
\hline Knotted1-like homeodomain protein & Os05g03880 & -1.6 \\
\hline Homeobox domain, putative & Os01g19700 & -1.8 \\
\hline Homeodomain leucine zipper protein & Os10g41230 & -2.6 \\
\hline Homeobox domain, putative & Os02g43330 & -2.7 \\
\hline Knotted1-type homeobox protein & Os03g51710 & -5.1 \\
\hline WOX11 protein & Os07g48560 & -3.1 \\
\hline Myb-related protein, putative & Os05g07010 & 6.1 \\
\hline Myb-like DNA-binding domain & Os01g64360 & 1.2 \\
\hline Myb-like DNA-binding domain & Os11g45740 & -1.2 \\
\hline Myb-like DNA-binding domain & Os02g42850 & -1.3 \\
\hline Myb-like DNA-binding domain & Os06g28630 & -1.4 \\
\hline Myb-like DNA-binding domain & Os07g48870 & -1.9 \\
\hline Myb-like DNA-binding domain & Os12g37690 & -2.3 \\
\hline NAM protein, putative & Os $10 g 33760$ & 1.4 \\
\hline OsNAC5 protein & Os05g34830 & -1.3 \\
\hline OsNAC5 protein & Os11g08210 & -1.3 \\
\hline No apical meristem (NAM) protein & Os07g48550 & -1.4 \\
\hline OsNAC1 protein & Os03g42630 & -1.5 \\
\hline No apical meristem (NAM) protein & Os12g03040 & -1.7 \\
\hline TCP family transcription factor & Os06g 12230 & 1.3 \\
\hline TCP family transcription factor & Os05g43760 & 1.2 \\
\hline WRKY DNA -binding domain & Os06g44010 & 1.3 \\
\hline WRKY DNA -binding domain & Os01g09100 & -2 \\
\hline \multicolumn{3}{|l|}{ Hormone response } \\
\hline Auxin efflux carrier & Os01g45550 & 1 \\
\hline Auxin efflux carrier & Os09g38130 & -1.6 \\
\hline Auxin transporter & Os06g12610 & -1.8 \\
\hline Auxin induced protein & Os09g37480 & 1.2 \\
\hline Auxin responsive protein & Os07g29310 & 1.7 \\
\hline GH3 auxin-responsive promoter & Os01g57610 & -1.3 \\
\hline GH3 auxin-responsive promoter & Os05g05180 & -1.4 \\
\hline IAA-amino acid conjugate hydrolase-like protein & Os06g47620 & -1.4 \\
\hline Indole-3-glycerol phosphate synthase & Os09g08130 & 1.7 \\
\hline Adenylate isopentenyltransferase, putative & Os03g59570 & -3.1 \\
\hline Ethylene responsive factor & Os05g06320 & -1.4 \\
\hline
\end{tabular}


Table 2 Differentially expressed genes identified by microarray analysis (continued)

\begin{tabular}{|c|c|c|}
\hline Coded protein/putative function & OGI $^{\mathrm{a}}$ & $\mathbf{E R}^{\mathrm{b}}$ \\
\hline \multicolumn{3}{|l|}{ Cytochrome P450 } \\
\hline Cytochrome P450 & Os04g10160 & 6.4 \\
\hline Cytochrome P450 & Os02g36110 & 5.1 \\
\hline Cytochrome P450 & Os02g36190 & 4.5 \\
\hline Cytochrome P450 kaurene oxidase & Os06g37300 & 3.8 \\
\hline Cytochrome P450 & Os10g30390 & 3 \\
\hline Cytochrome P450 & Os08g39730 & 2.7 \\
\hline Cytochrome P450 & Os02g36030 & 2.6 \\
\hline Cytochrome P450 & Os11g05380 & 2.6 \\
\hline Cytochrome P450 & Os07g11970 & 2.4 \\
\hline Cytochrome P450 & Os05g01120 & -1.2 \\
\hline Cytochrome P450 & Os05g12040 & -1.6 \\
\hline Cytochrome P450 & Os03g04650 & -2.1 \\
\hline \multicolumn{3}{|l|}{ Protein degradation } \\
\hline Ubiquitin-conjugating enzyme E2 & Os05g48390 & -1.3 \\
\hline F-box domain, putative & Os01g57920 & 5.1 \\
\hline F-box domain, putative & Os01g60920 & -1.5 \\
\hline
\end{tabular}

A

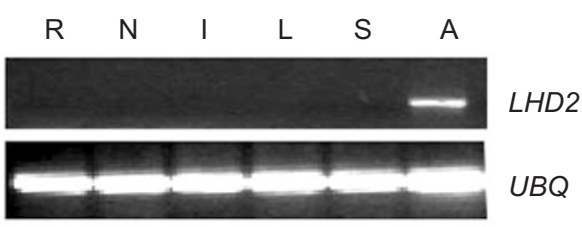

B

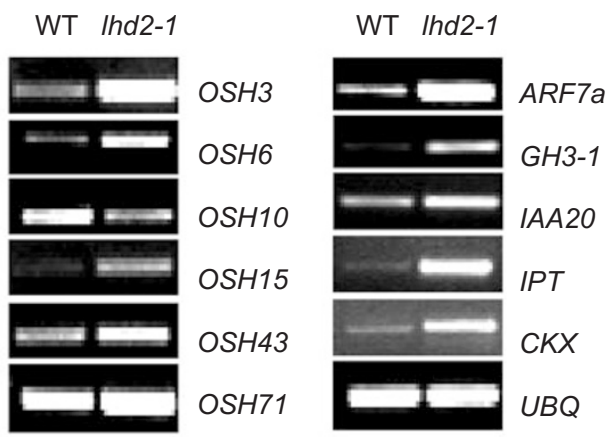

Figure 5 Expression of $L H D 2$ in organs and differentially expressed genes in $l h d 2-1$. (A) The LHD2 expression in organs. Total RNA was isolated from roots (R), nodes (N), internodes (I), leaves (L), leaf sheathes (S) and shoot apices (A) of wild-type plants. (B) Confirmation of differentially expressed genes in the $l$ hd 2 mutant plant. Amplification of ubiquitin cDNA was used to ensure that approximately equal amount of cDNA was loaded. regulating rice plastochron, we generated the genome-scale expression profile between wild-type and $l h d 2$ plants using AFFYMETRIX Rice Gene Chip (Santa Clara, USA). Classification analysis indicated that most of the differentially expressed genes in the mutant plants were related to transcription regulation, signal transduction and hormone response. The representative differential expression genes were summarized in Table 2. Among genes with significant alteration in expression, several genes related to the $K N O X$ signal pathway, cytokinin metabolism and auxin response were confirmed by RT-PCR analysis (Figure 5B). These results suggested that $L H D 2$ may regulate rice leaf initiation and stem elongation through KNOX genes and hormone related genes within the SAM. Further analysis is needed to dissect the regulatory pathway mediated by LHD2 in controlling shoot development of rice plant.

\section{Discussion}

The development of leaves, including the position, shape and size, is a major determinant factor that affects plant architecture. Phyllotaxy and plastochron are basic aspects of leaf development and are species specific. Mutants that affect either phyllotaxy or plastochron are useful tools to understand the mechanism of leaf formation. In case of 
rice, the study of phyllotaxy and plastochron is also of agronomic importance.

In this study, we have identified and characterized the rice mutant $l h d 2$ that exhibits defects in plastochron, stem elongation, tiller bud outgrowth and developmental phase transition. Molecular analysis revealed that $L H D 2$ is homologous to maize $T E 1$ that regulates the rate of leaf initiation in corn plants [20]. TE1 displays a significant similarity to Mei2, an RNA-binding protein from Schizosaccharomyces pombe that is required for both premeiotic DNA synthesis and the first reductional division of meiosis [29]. The similarity is highest in the three regions in both protein sequences that encode RNA-recognition motifs (RRMs). Previous studies on the sequences similarity demonstrated that this gene shares a much conserved exon-intron structure and a high degree of amino acid similarity among Poaceae species, especially a very high degree identity in conserved RRM motifs [20,21]. Our work showed that the mutation of LHD2 in rice can cause the similar phenotype to maize tel including shorten plastochron and reduced stem elongation, providing a strong evidence that $L H D 2$ is a functional orthologue of maize $T E 1$. However, unlike the maize tel mutant that exhibits defects in both plastochron and phyllotaxy, the rice $l h d 2$ mutant exclusively affects plastochron. Additionally, the phase transition from vegetative to reproductive growth is also suppressed in $l h d 2$, indicating the functional diversion of the TE1 family during the process of evolution.

The TE1 expression pattern in Poaceae species revealed by in situ hybridization suggested that $T E 1$ may involve in both leaf initiation and cell differentiation [21]. Our observation that stem elongation, phase transition and tiller bud outgrowth are all abnormal in $l h d 2$ is consistent with this hypothesis because cell differentiation contributes to all these developmental events. Although molecular genetic and cell biology approaches have made significant advances in understanding the regulatory mechanism in leaf formation, most researches have been focused on phyllotaxy due to the lack of mutants that are exclusively defective in plastochron. The rice $P L A 1$ gene is the first reported gene that is merely involved in regulating plastochron [17]. In view of the different performance between plal and $l h d 2$ on tiller number and developmental phase transition, we assumed that $L H D 2$ and $P L A 1$ may regulate rice plant leaf initiation temporally in a distinct genetic pathway.

Field inhibitory theory proposed that existing primordia use either biochemical or biophysical constraints to control phyllotaxy and plastochron [5]. However, the mechanism regulating plastochron is far to be elucidated. The comparison of genome-scale expression profile between wild-type and $l h d 2-1$ plants provided a clue that $L H D 2$ may regulate rice shoot development through KNOX and hormone- related genes. Further investigation will contribute to understand the action of LHD2 in controlling the shoot development of rice plants, which may also be conserved in grass species.

\section{Acknowledgments}

We thank Jiayi Xie (Institute of Microbiology, Chinese Academy of Sciences) and Ying Lan (Institute of Genetics and Developmental Biology, Chinese Academy of Sciences) for assistance in scanning electron microscopic observation. This work was supported by grants from National Natural Science Foundation of China (30330040 and 30221002).

\section{References}

1 Gallois JL, Woodward C, Reddy GV, Sablowski R. Combined SHOOT MERISTEMLESS and WUSCHEL trigger ectopic organogenesis in Arabidopsis. Development 2002; 129:32073217.

2 Golz JF, Hudson A. Signalling in plant lateral organ development. Plant Cell 2002; 14 Suppl:S277-S288.

3 Hake S, Smith HM, Holtan H, et al. The role of knox genes in plant development. Annu Rev Cell Dev Biol 2004; 20:125151.

4 Jasinski S, Piazza P, Craft J, et al. KNOX action in Arabidopsis is mediated by coordinate regulation of cytokinin and gibberellin activities. Curr Biol 2005; 15:1560-1565.

5 Fleming AJ. The control of leaf development. New Phytol 2005; 166:9-20.

6 Steeves TA, Sussex IM. eds. Patterns in plant development. $2^{\text {nd }}$ Edition. New York: Cambridge University Press, 1989.

7 Fleming AJ. Formation of primordia and phyllotaxy. Curr Opin Plant Biol 2005; 8:53-58.

8 Reinhardt D, Mandel T, Kuhlemeier C. Auxin regulates the initiation and radial position of plant lateral organs. Plant Cell 2000; 12:507-518.

9 Benkova E, Michniewicz M, Sauer M, et al. Local, efflux-dependent auxin gradients as a common module for plant organ formation. Cell 2003; 115:591-602.

10 Reinhardt D, Pesce ER, Stieger P, et al. Regulation of phyllotaxis by polar auxin transport. Nature 2003; 426:255-260.

11 Chaudhury AM, Letham S, Craig S, Dennis ES. amp1 - a mutant with high cytokinin levels and altered embryonic pattern, faster vegetative growth, constitutive photomorphogenesis and precocious flowering. Plant J 1993; 4:907-916.

12 Itoh JI, Hasegawa A, Kitano H, Nagato Y. A recessive heterochronic mutation, plastochron1, shortens the plastochron and elongates the vegetative phase in rice. Plant Cell 1998; 10:15111522 .

13 Jackson D, Hake S. Control of phyllotaxy in maize by the abphyll gene. Development 1999; 126:315-323.

14 Itoh JI, Kitano H, Matsuoka M, Nagato Y. Shoot organization genes regulate shoot apical meristem organization and the pattern of leaf primordium initiation in rice. Plant Cell 2000; 12:21612174 . 
15 Giulini A, Wang J, Jackson D. Control of phyllotaxy by the cytokinin-inducible response regulator homologue ABPHYL1. Nature 2004; 430:1031-1034.

16 Scanlon MJ. Force fields and phyllotaxy: an old model comes of age. Trends Plant Sci 1998; 3:413-414.

17 Miyoshi K, Ahn BO, Kawakatsu T, et al. PLASTOCHRON1, a timekeeper of leaf initiation in rice, encodes cytochrome $\mathrm{P} 450$. Proc Natl Acad Sci USA 2004; 101:875-880.

18 Maksymowych R, Cordero RE, Erickson RO. Long-term developmental changes in Xanthium induced by gibberellic acid. Amer J Bot. 1976; 63:1047-1053.

19 Scanlon MJ. The polar auxin transport inhibitor N-1-naphthylphthalamic acid disrupts leaf initiation, KNOX protein regulation, and formation of leaf margins in maize. Plant Physiol 2003; 133:597-605.

20 Veit B, Briggs SP, Schmidt RJ, Yanofsky MF, Hake S. Regulation of leaf initiation by the terminal ear 1 gene of maize. Nature 1998; 393:166-168.

21 Paquet N, Bernadet M, Morin H, et al. Expression patterns of TEL genes in Poaceae suggest a conserved association with cell differentiation. J Exp Bot 2005; 56:1605-1614.

$22 \mathrm{Li} \mathrm{X}$, Qian Q, Fu Z, et al. Control of tillering in rice. Nature $2003 ;$ 422:618-621.
23 Sakamoto T, Matsuoka M. Generating high-yielding varieties by genetic manipulation of plant architecture. Curr Opin Biotechnol 2004; 15:144-147.

24 Wang Y, Li J. The plant architecture of rice (Oryza sativa). Plant Mol Biol 2005; 59:75-84.

$25 \mathrm{Hu}$ YH. An X-ray induced panicle-degenerating mutant in rice. Jap J Breed 1961; 11:19-23.

26 Duan YL, Wu WR, Liu HQ, et al. Genetic analysis and gene mapping of leafy head (lhd), a mutant blocking the differentiation of rachis branches in rice (Oryza sativa L.). Chinese Sci Bull 2003; 48:2201-2205.

$27 \mathrm{Hu}$ Y, Bao F, Li J. Promotive effect of brassinosteroids on cell division involves a distinct $C y c D 3$-induction pathway in Arabidopsis. Plant J 2000; 24:693-701.

28 Mou Z, He Y, Dai Y, Liu X, Li J. Deficiency in fatty acid synthase leads to premature cell death and dramatic alterations in plant morphology. Plant Cell 2000; 12:405-418.

29 Watanabe Y, Lino Y, Furuhata K, Shimoda C, Yamamoto M. The S.pombe mei2 gene encoding a crucial molecule for commitment to meiosis is under the regulation of cAMP. EMBO J 1988; 7:761-767.

Edited by Lu Liang 